\title{
The semantic view, if plausible, is syntactic
}

\author{
Hans Halvorson*
}

March 13, 2013

\begin{abstract}
Halvorson (2012) argues that the semantic view of theories leads to absurdities. Glymour (2013) shows how to inoculate the semantic view against Halvorson's criticisms, namely by making it into a syntactic view of theories. I argue that this modified semantic-syntactic view cannot do the philosophical work that the original "languagefree" semantic view was supposed to do.
\end{abstract}

According to Clark Glymour (2013), my argument against the semantic view of theories omits an important piece of information - namely, the model-theoretic account of theoretical equivalence given by de Bouvere (1965). I plead guilty to this charge. But, as Glymour points out, for a semanticist to avail herself of de Bouvere's account, she must put language back into her account of theories.

I claim that if we add language to the semantic view, then we have a syntactic view, indistinguishable from the oft-reviled "received view of theories." Let's call this modified view "semantic $+\mathrm{L}$ ". But if semantic $+\mathrm{L}$ is just the syntactic view, then the following sorts of claims are shown to be nonsense:

A certain view $\phi$ about scientific theories is not plausible if one is thinking in terms of the syntactic view of theories. But if one adopts the semantic view, then $\phi$ proves to be defensible.

Such claims are abundant in the philosophy of science literature. For example, van Fraassen has frequently claimed that earlier attempts at an empiricist philosophy of science were scuttled by the syntactic view of theories; but the semantic view allows for the consistent development of constructive empiricism. If Glymour is correct that the semantic view collapses into the syntactic view, then van Fraassen's rhetoric cannot be maintained. Similarly,

\footnotetext{
*Department of Philosophy, Princeton University. hhalvors@princeton.edu
} 
recent work on structural realism seems to derive plausibility, in part, from the semantic view of theories. But if the semantic view collapses into the syntactic view, then structural realism is no more plausible today than it was in the time of Carnap and Hempel.

Glymour shows that the arguments in (Halvorson, 2012) are ineffective against semantic + L. That may be true; but those arguments were directed against a stronger version of the semantic view of theories - a version that takes seriously van Fraassen's claim that, "the impact of Suppes' innovation is lost if models are defined, as in many standard logic texts, to be partially linguistic entities, each yoked to a particular syntax..." van Fraassen, 1989, 366). Call this view "semantic-L." Halvorson (2012) argues that semantic-L leads to absurdity, and Glymour cedes this point. Of course, it's a dialectical challenge to show that a partially articulated position (e.g. semantic-L) leads to absurdity, because articulating it requires one to say some absurd things. I attempted, in particular, to equip semantic-L with an account of when two models of a theory are isomorphic. (Recall that such an account cannot make reference to language.) Glymour articulates the notion that I implicitly employed, calling it $\mathrm{H}$-isomorphism:

Two structures $\left\langle S, R_{1}, \ldots, R_{n}\right\rangle$ and $\left\langle S^{\prime}, R_{1}^{\prime}, \ldots, R_{n}^{\prime}\right\rangle$ are $H$-isomorphic just in case there is a bijection $j: S \rightarrow S^{\prime}$ such that $\left\langle a_{1}, \ldots, a_{n}\right\rangle \in R_{i}$ if and only if $\left\langle j\left(a_{1}\right), \ldots, j\left(a_{n}\right)\right\rangle \in R_{i}^{\prime}$.

Glymour argues that H-isomorphism is far too liberal, for it would even allow that elementarily inequivalent models are $\mathrm{H}$-isomorphic. I completely agree! I was trying to show that the semantic-L view leads to absurdities, and semantic-L has no other notion of isomorphism between models besides H-isomorphism. In other words, semantic-L needs H-isomorphism, and H-isomorphism leads to absurdity. Glymour and I agree that semantic-L leads to absurdity.

Thus, Glymour and I agree that the only plausible semantic view is semantic+L. But I'm not sure that Glymour appreciates that adding the "L" undermines some of the more philosophically interesting claims made by proponents of the semantic view of theories. Consider, in particular, the claim that a class of models is the invariant content behind different syntactic presentations of a theory.

"Theories are extralinguistic entities which may be described or characterized by a number of different linguistic formulations." (Suppe, 1977, 221)

"While a theory may have many different formulations, its set of models is what is important." (van Fraassen, 2008, 309)

Glymour suggests that semantic+L maintains this picture of a class of models as the "invariant content" among different linguistic formulations of a theory. He says: 
"To be at all plausible, the semantic view must distinguish between the content of a theory - its class of models - and the means of characterizing that class — the theory expressed in some language." (pp. 4-5)

"A tenable semantic view, as I construe it, also requires that there is a model theoretic relation that establishes a common content to different presentations of a theory in different languages." (p. 6)

But semantic $+\mathrm{L}$ is inconsistent with the idea that a class of models is the invariant content of a theory. In "Theories versus formulations" (pp. 201-203 of Halvorson 2012), I point out that different formulations of the same theory have different classes of models. For example, the theory of groups has formulations in several different languages including $L_{0}=\{0\}$, $L_{1}=\left\{\circ,{ }^{-1}\right\}, L_{2}=\{\circ, e\}$ or $L_{3}=\left\{\circ,{ }^{-1}, e\right\}$. All of these formulations of group theory are equivalent; but they all have different classes of models. Indeed, there is no single class of models that has preferential status as "the" class of groups. Therefore, the class of models is not the common content of different formulations of the same theory.

So, I mean to make a stronger point than Glymour does. Glymour claims that language is necessary for presenting theoretical content:

"Like it or not, on the semantic view language and logical syntax are indispensable tools for the presentation of theoretical content ..." (p. 5)

But what is the theoretical content that the language is supposed to be presenting? As we saw above, the shared content cannot be a class of models - because different languages do not share models in common. Semanticists, and perhaps Glymour too, may have been misled by a false analogy to geometric invariants. For example, a manifold $M$ may be presented via several distinct (global) coordinate charts. But these various coordinate charts all describe the same manifold. Semanticists may have thought that a class of models can play the same role relative to the linguistic formulations of a theory that a manifold plays relative to its various coordinate charts. But this analogy fails: different linguistic formulations of a theory cannot describe the same class of models.

Recall that, by definition, a model is a mapping from a language to a set-theoretic structure 1 . Just as the definition of a function includes a specification of its domain, the definition of a model includes a specification of its language. Accordingly, reducing a model to a bare set-theoretic $n$-tuple would be akin to reducing a function $f: A \rightarrow B$ to its image

\footnotetext{
${ }^{1}$ I find it most illuminating to think of models as functors. If $T$ is a first-order theory, then models of $T$ correspond to functors from the syntactic category $C_{T}$ to the category of sets. See (Makkai and Reyes, 1977).
} 
$f(A)$. Obviously, the function $f$ contains more data than its image $f(A)$; and in exactly the same way, a model contains more data than just the resulting set-theoretic structure.

So, models are necessarily language-bound objects. But this is not to say that theories in different languages are necessarily inequivalent. Rather, as in Figure 4 of (Halvorson, 2012), there is an interesting equivalence relation between theories formulated in different languages, and also a corresponding equivalence relation between classes of models. For classes of models, the relevant equivalence relation happens to be that given by de Bouvere's 1965 account of synonymy ${ }^{2}$

This point (that models are not the invariant content of equivalent theories) raises a problem for structural realism. Recall that some structural realists want to subscribe to the view that:

(P1) To believe a theory $T$ means to believe that the world is isomorphic to a model of $T$.

But structural realists should also be committed to the claim that:

(P2) There is no good reason to believe $T$ over an equivalent theory $T^{\prime}$.

(If they disagree with this claim, then we have given the wrong account of equivalence!) But P1 and P2 do not play nicely together. Together they entail that believing $T$ means believing that the world is isomorphic to a model of $T$, but also that the world is isomorphic to a model of $T^{\prime}$. But nothing is isomorphic both to a model of $T$ and to a model of $T^{\prime}$. So which are we supposed to believe: that the world is isomorphic to a model of $T$, or that the world is isomorphic to a model of $T^{\prime}$ ? It seems that structural realism would make Buridan's asses out of all of us.

Acknowledgments: These ideas were sharpened in conversations with Jim Weatherall.

\section{References}

de Bouvere, K. (1965). Synonymous theories. In J. Addison et al. (Eds.), The theory of models, pp. 402-406. Amsterdam: North Holland.

Glymour, C. (2013). Theoretical equivalence and the semantic view of theories. Philosophy of Science. this issue.

\footnotetext{
${ }^{2} \ldots$ at least in simple cases. de Bouvere's account works only for single-sorted theories, and it doesn't allow multi-dimensional interpretations as in (Hodges, 1993 , Chap. 5).
} 
Halvorson, H. (2012). What scientific theories could not be. Philosophy of Science 79(2), $183-206$.

Hodges, W. (1993). Model theory. Cambridge University Press.

Makkai, M. and G. E. Reyes (1977). First order categorical logic. Springer-Verlag.

Suppe, F. (1977). The structure of scientific theories. University of Illinois Press.

van Fraassen, B. C. (1989). Laws and symmetry. Oxford University Press.

van Fraassen, B. C. (2008). Scientific representation. Oxford University Press. 